\title{
First report of tumor treating fields use in combination with bevacizumab in a pediatric patient: a case report
}

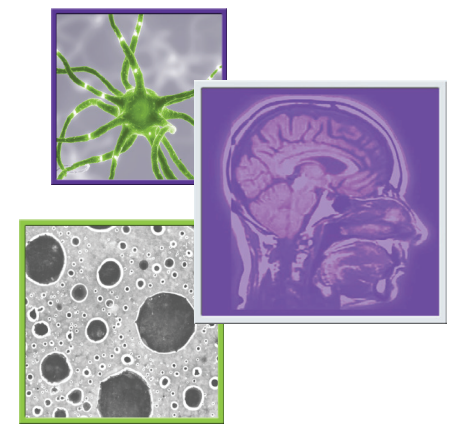

\author{
Daniel O'Connell ${ }^{*, 1}$, Violet Shen', William Loudon' \& Daniela A Bota'
}

\section{Practice points}

- Although only representing $15-20 \%$ of pediatric CNS tumors, $70-90 \%$ of children with high-grade glioma are dead within 2 years of diagnosis.

- Given the unclear nature of temozolomide in the treatment of pediatric high-grade glioma, the need for further studies both using and not using temozolomide in conjunction with an alternative regimen such as tumor treating fields (TTF) becomes apparent.

- TTF involve intermediate-frequency, low-intensity alternating electrical fields that selectively kill or arrest the growth of rapidly dividing cancer cells during mitosis.

- In the adult population, significantly prolonged progression-free and overall survival in glioblastoma patients who had TTF added to maintenance temozolomide chemotherapy has been demonstrated.

- TTF appear to have forestalled the growth of recurrent glioblastoma in our patient for 7 months.

- The dermatologic side effects of the TTF device experienced by our patient were minimal.

- Clinical trials to further validate the effect of TTF on high-grade glioma in conjunction with molecular analyses of baseline tumor biopsies in adults and children are urgently needed in the coming years.

We report the first case of a pediatric patient with glioblastoma (GBM; WHO grade IV astrocytoma) successfully treated with tumor treating fields (TTF). The patient was diagnosed with GBM when 13 years of age and progressed through surgical resection, radiotherapy and chemotherapy. Discrete tumor growth visualized on MRI with stable neurological examination was monitored for 6 months with subsequent stable disease observed radiographically and clinically for 7 months while adherent to Optune ${ }^{\circledast}$ (TTF). TTF thereby played a role in forestalling recurrent GBM growth in this young woman for 7 months without significant adverse effects. We propose that TTF therapy is a potential valuable treatment in this small, but sick, patient population.

First draft submitted: 17 March 2016; Accepted for publication: 30 August 2016; Published online: 5 December 2016

\section{Presentation}

A 13-year-old female patient presented with progressively worsening headaches. The symptoms were initially attributed to having the common cold or allergies. She was living with her parents and attending middle school at the time, a nonsmoker and a nondrinker. She was receiving A and $\mathrm{B}$ grades while leading an active social life. There was a strong family history of colon cancer on the maternal side. The patient's vital statistics included a height of $164 \mathrm{~cm}$, a weight of

'Department of Neurology, University of California, Irvine Medical Center, Orange, CA, USA

*Author for correspondence: Tel.: +1 310825 5321; Fax: +1 310825 0644; DanielOConnell@mednet.ucla.edu 


\section{KEYWORDS}

- astrocytoma

- bevacizumab • cisplatin

- CNS neoplasm

- glioblastoma • glioma

- pediatric • recurrence

- temozolomide • tumor treating fields
$60 \mathrm{~kg}$ and a BMI of 22. Café au lait spots were notably absent. Karnofsky performance score consistently rated $80 \%$ or above during assessments. On neurological examination, she had occasional difficulty performing serial sevens, experienced fluctuating concentration and ataxic gait, but was otherwise within normal limits. A month after the onset of headaches, her primary care provider ordered a head computed tomography which suggested the presence of a rightsided supratentorial brain tumor. MRI showed both areas of contrast enhancement and cystic necrosis (see Figure 1). She was referred to the local children's hospital where she had her first resection.

\section{Pathology \& diagnosis}

Pathology was reviewed at Mayo Clinic and Johns Hopkins Reference Laboratories documenting WHO grade IV astrocytoma (glioblastoma $[\mathrm{GBM}]$ ) with absent IDH1 mutation, positive GFAP, positive S100, positive OLIG2 co-expression and, to a lesser extent, positive synaptophysin. $B R A F$ mutation was not detected. The tumor, composed of cells with largely irregular, hyperchromatic nuclei and relatively abundant cytoplasm, was highly infiltrative and mitotically active ( $>30$ mitoses per 10 High Power Fields). Microcysts, hyperplastic microvasculature and necrosis were present. Despite the relatively strong synaptophysin expression, the morphology of the specimen was atypical for primitive neuroectodermal tumor (PNET). Features most suggestive of PNET still fell short of the classic PNET-like component of glial tumors and staining characteristics of the tumor remained constant from this partially suggestive area to the surrounding tumor. Immunohistochemical stains were performed on paraffin-embedded tissue also using antibodies to $\mathrm{p} 53$ protein, $\mathrm{Ki}-67$ and $\mathrm{KP} 1$. Overexpression of $\mathrm{p} 53$ protein in most tumor cells highlighted both the more cellular and the infiltrative component of the tumor. Ki-67/MIB1 labeling was brisk with a proliferative index up to $50-60 \%$ in the most solid area of the tumor highlighting the proliferative activity of the infiltrative cells. CD68/KP1 was positive in microglia, scant macrophages well distinct from tumor cells. These immunohistochemical stains further supported the diagnosis of GBM (see Figure 2 for corresponding pathology). Tissue collected from the second resection reaffirmed this consensus. The clinicopathologic and genetic features of the current series of rare brain tumors with GBM and PNET features support the concept of an underlying high-grade glioma with secondary development of PNET-like foci [1]. Recent literature further suggests via concordant DNA methylation profiling that many CNS PNETs can reliably be separated and reclassified into high-grade glioma reference subgroups based on embryonal histology and molecular analysis [2] In most cases, clinical presentation of these morphological variants is identical and patient survival is short not differing significantly from standard GBM.

\section{Treatment}

The patient subsequently received a course of craniospinal radiation at $36.0 \mathrm{~Gy}$ in 20 fractions. The metastasis around the falx received
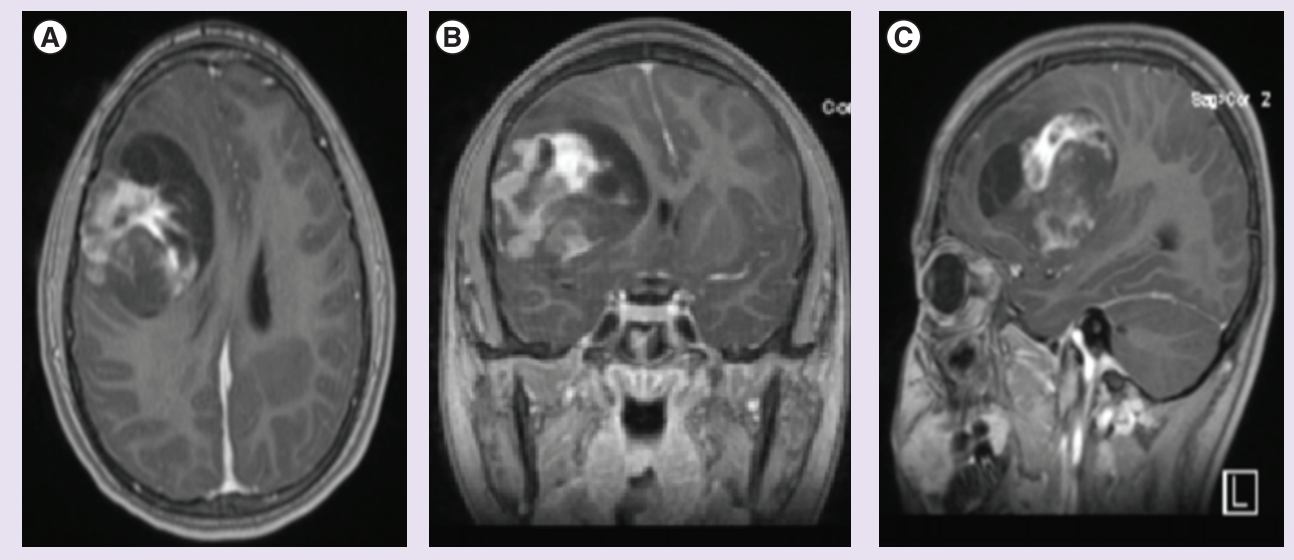

Figure 1. Preoperative MRI brain images at the time of tumor diagnosis. (A) T1 postcontrast axial, (B) T1 postcontrast coronal, (C) T1 postcontrast sagittal. 


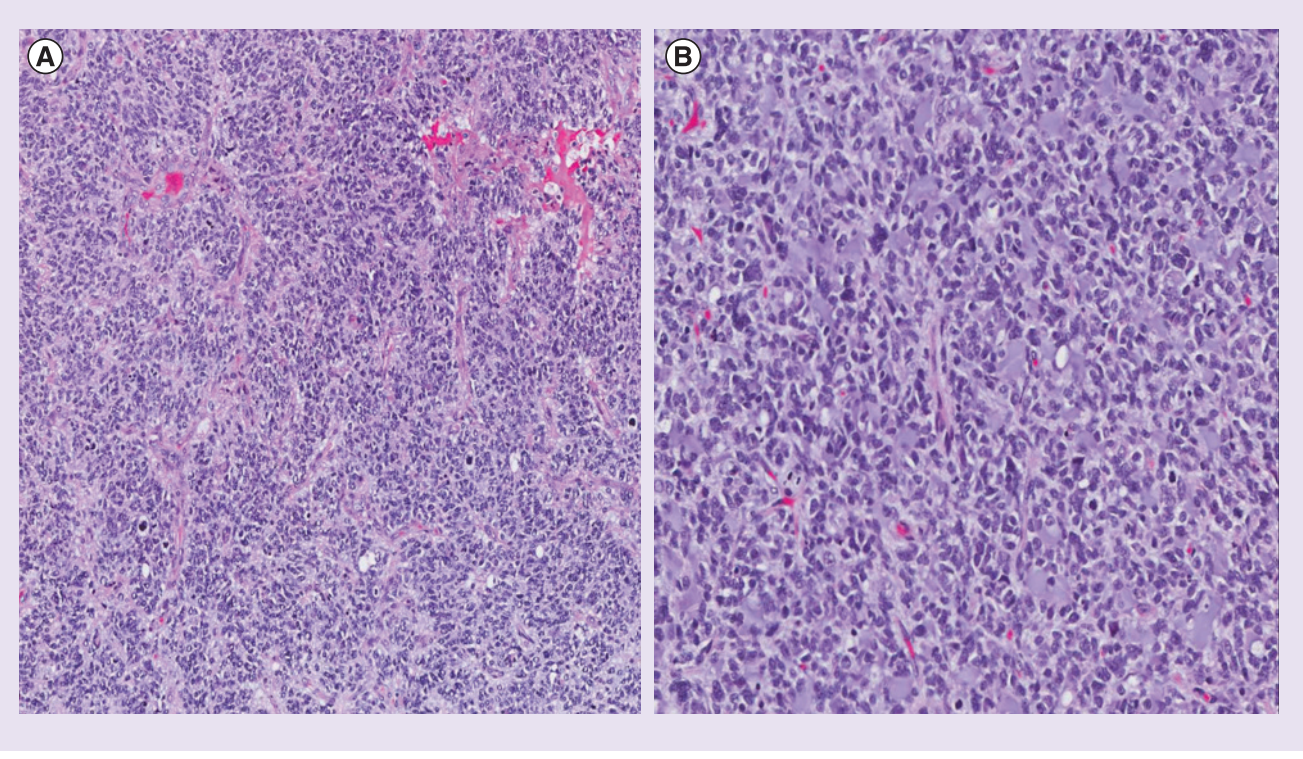

Figure 2. Initial brain pathology. (A) A hematoxylin-eosin-stained section of cortical tissue with microvascular proliferation; areas of necrosis present but not visualized here (objective $\times 10$ ).

(B) A hematoxylin-eosin-stained section of cortical tissue with pronounced hypercellularity, nuclear pleomorphism and mitotic figures easily identified (objective $\times 20$ ).

50.4 Gy in 28 fractions. The right-sided frontal lobe tumor received a total of $69.4 \mathrm{~Gy}$ in 33 fractions. The patient also received concomitant temozolomide $90 \mathrm{mg} / \mathrm{msq}$ daily for 42 days based upon the ACNS0822 protocol followed by a 4-week rest period [3]. Consolidation treatment further included cisplatin at $75 \mathrm{mg} / \mathrm{msq}$ on day 1 and temozolomide $150 \mathrm{mg} / \mathrm{msq}$ on days $2-6$ in 28 -day cycles for approximately 12 cycles with dose reductions during the last cycles secondary to hematological toxicities attributed to cisplatin. She received a total of seven doses of cisplatin with a cumulative dose of $525 \mathrm{mg} / \mathrm{msq}$. Renal function was preserved and audiogram showed minimal hearing loss. Given lack of enrollment at the time in a clinical trial, the patient was maintained on $150 \mathrm{mg} / \mathrm{msq}$ adjuvant temozolomide administered five times per month given high risk of progression and followed closely. Seizures began to recur and her repeat MRI showed further disease progression 20 months after initial diagnosis. This progression was reflected by two foci of nodular contrast enhancement, one dural based measuring $5.6 \mathrm{~mm}$ at the anterosuperior margin of the operative bed, and the other more linear measuring $5.3 \mathrm{~mm}$ in the central deep white matter of the right frontal lobe. In addition, there was new T2 signal abnormality. She underwent stereotactic-guided right frontoparietal craniotomy and near total resection ( $\geq 90 \%$ resected) of the right frontal lobe with residual rim of abnormal signal intensity noted.

The patient then came for a second opinion at a comprehensive brain tumor program. Optune $^{\circledR}$ (Novocure, NH, USA) plus bevacizumab was decided upon as a combined treatment regimen based upon Patient Registry Dataset (PRiDe) data given recurrence of her neoplasm [4]. Approximately 5 weeks after surgery, the patient was started on bevacizumab $10 \mathrm{mg} / \mathrm{kg}$ administered every 2 weeks. The Optune system was initiated 6 weeks after starting bevacizumab (see Figure 3 for the MRI performed shortly after starting bevacizumab and tumor treating fields [TTF]). The device was set at the standard adult frequency of $200 \mathrm{kHz}$, energy of $0.7 \mathrm{~V} / \mathrm{cm}$ with the rightsided frontal lobe lesion as the primary target. Treatment course consisted of 4-week cycles with maximal adherence strongly encouraged (24 h/day). Our patient successfully maintained an acceptable level of adherence $(\geq 75 \%$ per day) to the device per monthly report cards sent to clinic by wearing the device at home and at school [5]. Bevacizumab was continued along with TTF for the whole duration of therapy until the time of further progression (13 months). 


\section{Outcome}

She was assessed on a monthly basis by history taking, neurological examination, laboratory and every other month MRI studies. The side effects experienced by the patient during this period were minimal. She reported some mild scalp irritation and skin breakdown for which she was prescribed over-the-counter $1 \%$ hydrocortisone cream as needed [6]. The issue resolved at the time of her next appointment. She continued her usual activities. Brain MRI with and without contrast showed stable disease from 6 months after starting TTF to almost 7 months later. MRI then demonstrated progression according to 2010 Response Assessment in Neuro-Oncology criteria (see Figure 4 showing increased T2 hyperintensity in the right basal ganglia and temporal lobe reflecting nonenhancing tumor recurrence) [7]. The patient was able to complete all of the activities appropriate for a child of her age through the time of further progression. After progression, Optune was stopped and she was continued on bevacizumab. She later underwent a FoundationOne ${ }^{\circledR}$ (MA, USA) next-generation sequencing-based assay for the purpose of GBM-related therapeutic implication. She was started crizotinib due to having a ROS1 mutation and continued to be followed in clinic [8].

\section{Discussion \& conclusion}

High-grade gliomas in the young (WHO grades III and IV) are uncommon, with incidence rates of 0.08 for anaplastic astrocytoma and 0.14 for GBM per 100,000 persons [9]. Nevertheless, they carry with them a devastating prognosis, especially when involving the brainstem, with patients seldom achieving long-term survival. There are even worse prognoses and fewer options for children with recurrent high-grade glioma. Although only representing $15-20 \%$ of pediatric CNS tumors, 70-90\% of these highgrade glioma patients are dead within 2 years of diagnosis [10]. Although initial gross total or near total resection has demonstrated increased progression-free survival per Children's Cancer Group (CCG) Study CCG-945, and focal radiation therapy has been a mainstay in children
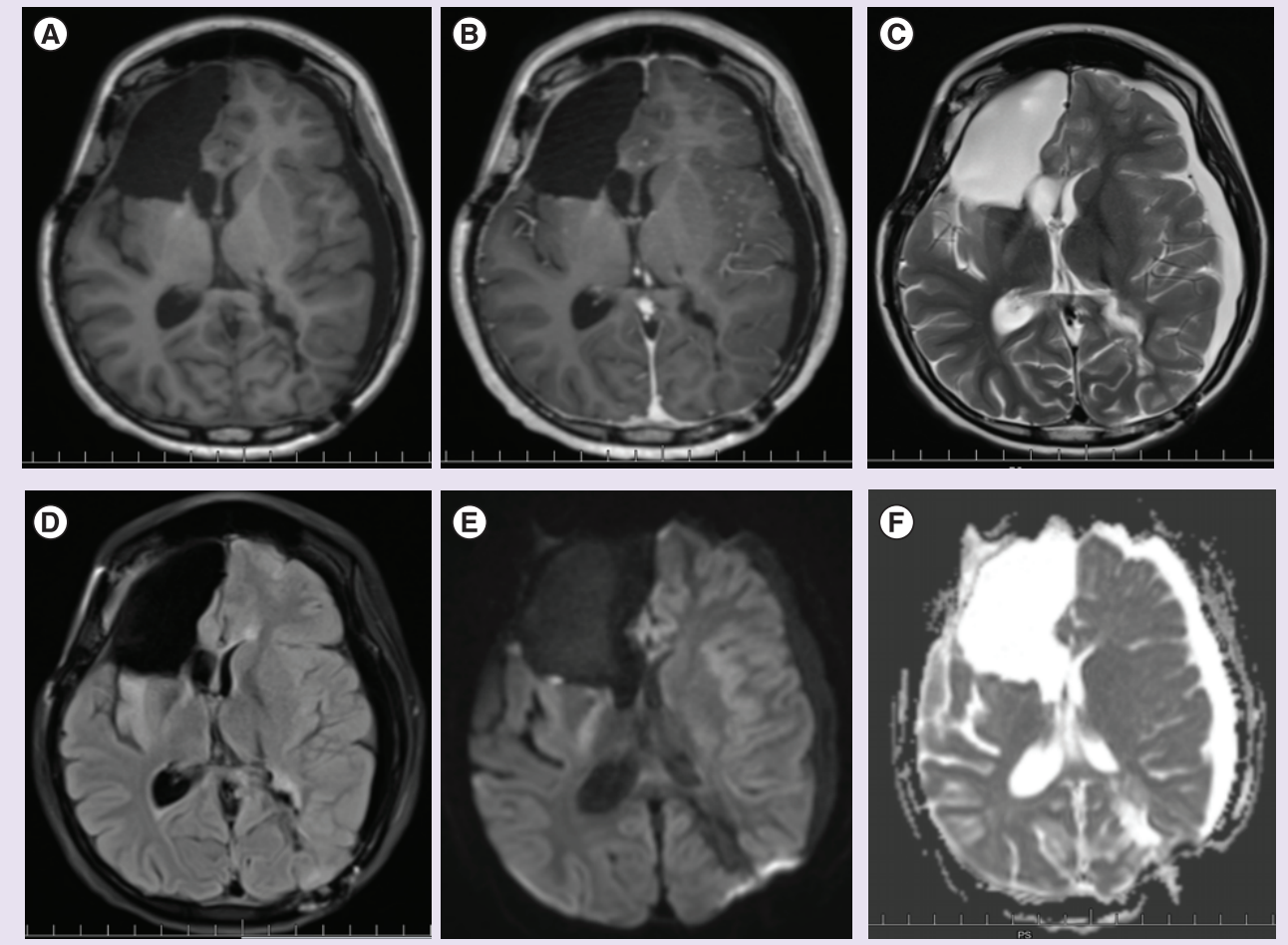

Figure 3. Postoperative MRI brain images shortly after starting bevacizumab and tumor treating fields. (A) T1 precontrast axial. (B) T1 postcontrast axial. (C) T2 axial. (D) T2 fluid attenuated inversion recovery axial. (E) Diffusion weighted b1000 axial. (F) Corresponding apparent diffusion coefficient axial. 

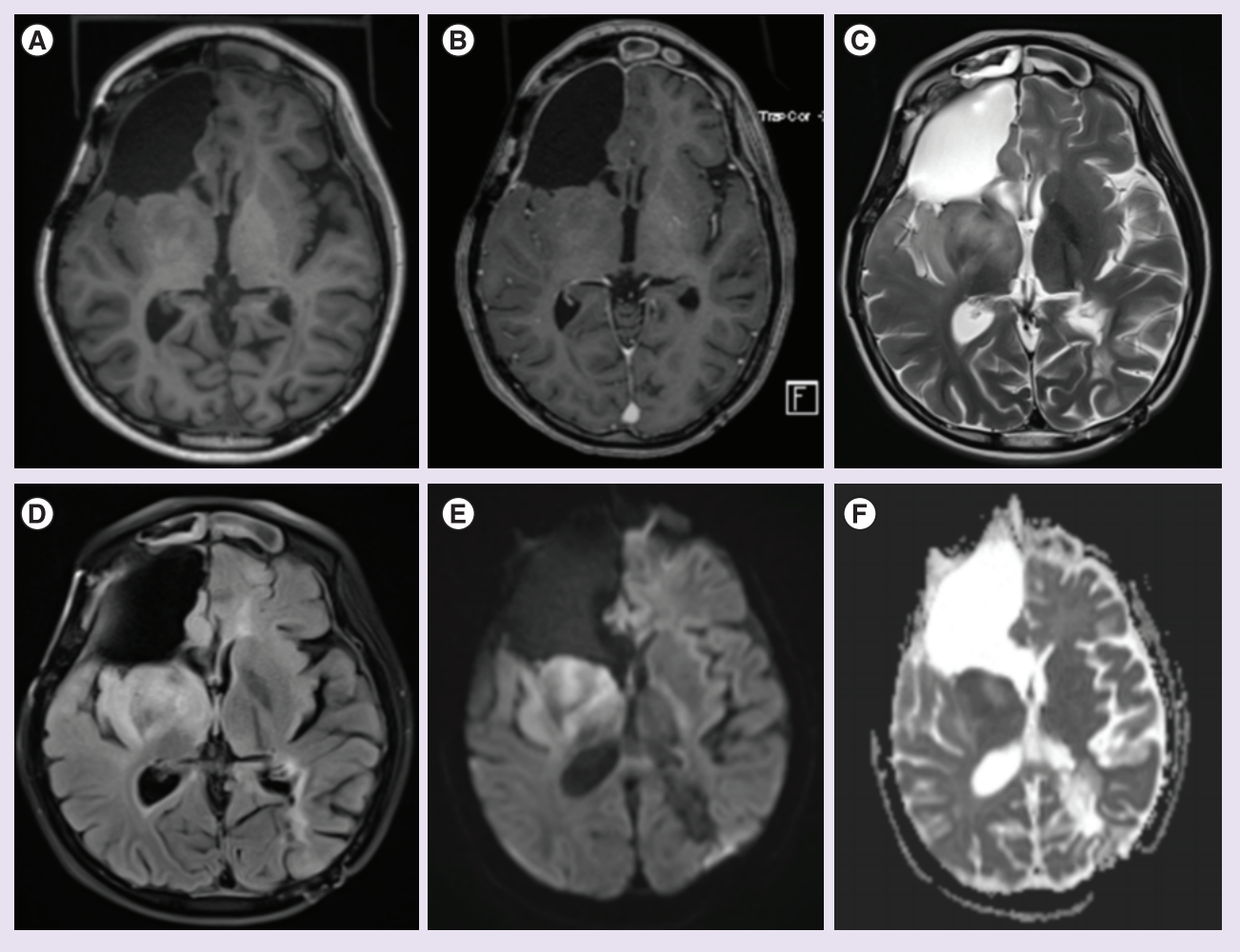

Figure 4. Postoperative MRI brain images at the time of tumor progression. (A) T1 precontrast axial. (B) T1 postcontrast axial. (C) T2 axial. (D) T2 fluid attenuated inversion recovery axial. (E) Diffusion-weighted b1000 axial. (F) Corresponding apparent diffusion coefficient axial

greater than 3 years of age, there is currently no accepted standard of care regarding chemotherapies, their doses and durations for pediatric patients with high-grade glioma. Treatment algorithms are based upon therapies approved for adult populations [11]. The standard of care in adults with GBM consists of initial gross total resection followed by focal irradiation, concomitant chemotherapy and then adjuvant chemotherapy in the form of temozolomide. At the time of recurrence, only bevacizumab and the Optune device have US FDA approval in the treatment of adult GBM. Therefore, many of the adult patients actively participate in clinical trials after exhausting their FDA approved therapies.

The approved options for children and teenagers with GBM are even more limited. As of now, neither temozolomide nor bevacizumab is formally approved by the FDA for pediatric gliomas. Hyperfractionated and hypofractionated ionizing radiotherapy techniques have not been proven to result in significant benefit. The
CCG sponsored CCG-943 trial in 1989 first demonstrated improved survival in pediatric GBM cases with the addition of chemotherapy to radiation therapy at least negating any further trial randomization to radiotherapy alone but without supporting any particular chemotherapeutic agent as first line [12]. The later crossover to temozolomide as chemotherapy of choice in the pediatric high-grade glioma population reflects the 2005 data published by Stupp et al. demonstrating the benefit of temozolomide in addition to radiation therapy versus radiotherapy alone [13]. Bevacizumab is often used along with temozolomide for maintenance therapy following the accepted adult model for tumor recurrence [14]. However, a recent feasibility and Phase II trial demonstrated that neither bevacizumab nor vorinostat proved superior to temozolomide as a radiosensitizer in pediatric high-grade glioma [15]. Fortunately, in our patient's case, there is evidence to suggest that CNS tumors in the young with combined features of malignant glioma and PNET, although rare and poorly characterized, 
may ultimately respond better to platinum-based chemotherapy such as cisplatin than to temozolomide [16]. The relative inefficacy of temozolomide in the treatment of pediatric high-grade glioma has even led some to question using it to treat these patients given its negative implications. The consistent adjuvant dosing of $150 \mathrm{mg} / \mathrm{msq} /$ day temozolomide was considered appropriate in this patient given the failure of maintenance temozolomide dosed at $200 \mathrm{mg} / \mathrm{msq} /$ day for 5 days in 28-day cycles following the first month of adjuvant chemotherapy in the pediatric population to demonstrate either overall improvement in patient outcome or noninferiority as shown in a single-arm Phase II trial, ACNS0126 [17]. Given the unclear benefit of temozolomide in the treatment of pediatric high-grade glioma, the need for further studies, both using and not using temozolomide in conjunction with an alternative regimen such as Optune, becomes apparent.

The use of TTF in cases of pediatric highgrade glioma remains a novel suggestion with no clinical trials currently in progress. Optune is an approved antimitotic, nonionizing radiation treatment for adult ( $>18$ years of age) patients with recurrent GBM. A 2009 study of chemotherapeutic treatment combined with TTF in vitro, in vivo and in a pilot clinical trial demonstrated that chemotherapeutic efficacy and sensitivity can increase without increasing treatment-related toxicity [18]. A pivotal 2012 study then demonstrated that when compared with physician's choice chemotherapy in adult patients with recurrent GBM, TTF therapy can obtain a similar median overall survival with significantly fewer adverse effects (6 vs $16 \%, \mathrm{p}=0.022$ ) and no decrement in quality of life relative to conventional chemotherapies [19]. Even more recently, Stupp et al. demonstrated significantly prolonged progression-free and overall survival in GBM patients who had TTF added to maintenance temozolomide chemotherapy following standardized chemoradiation therapy further elucidating its benefit in the management of high-grade glioma [20].

The presumptive mechanism of TTF involves intermediate-frequency, low-intensity alternating electrical fields selectively killing or arresting the growth of rapidly dividing cancer cells. This effect is thought to be accomplished by inhibiting the proper formation of the mitotic spindle and causing rapid membrane breakdown during cytokinesis [21]. Optune is believed to treat GBM by emitting alternating electric fields or TTF in a specified kilohertz range. The cancer cells are subject to alternating electric fields in the frequency of 100-250 kHz likely resulting in their cytoskeleton becoming disrupted, specifically during mitosis in the metaphase-to-anaphase transition, eventually causing blebbing and apoptosis [22]. The physical and electrical properties of cancer cells differ from cells that proliferate normally; cancer cells exhibit a lower-membrane electrical potential compared with normal proliferating cells and increased membrane fluidity affecting ease of deformation probably making them more susceptible to TTF [23].

The minimally accepted treatment duration for TTF therapy to reverse GBM tumor growth is 4 weeks with a period of 28 days considered one full treatment course [24]. Generally speaking, tumor responses to TTF develop slowly, as predicted by tumor volume kinetics models with a median time to response of 5.2 months [25]. This matches well with our patient who had discrete tumor growth visualized on MRI for 6 months prior to achieving stable disease. Aside from the skin irritation associated with the device, it does not appear from this case that any other significant side effect was withstood by the patient secondary to its use to achieve this stability at 6 months' time. TTF further played a role in forestalling recurrent GBM growth in this young woman for 7 additional months without significant adverse effects. The possible benefit of repeat resection was deferred given the lack of clinical and radiographic deterioration justifying further surgery at the time of TTF completion.

There was initial concern about using the Optune device on a young patient still in development. Devices like Optune are yet to be studied in pediatric patients. Theoretically, given its physical mechanism of action at the cellular level targeted within the brain, the use of TTF in the young should not differ significantly than in the adult population. Aside from the dentate gyrus of the hippocampus and the granular cells of the olfactory bulb migrating from the subventricular zone, the neuronal cells of the mammalian CNS cease to experience neurogenesis beyond the period of early development unlike the aberrant glial cells targeted by TTF [26]. Adult and pediatric patients alike can be considered remote from the period of neuronal differentiation during and shortly after embryogenesis. The effect of TTF upon mature astrocytes and other neuroglia in adolescents would be expected to parallel that of 
adults. There are no reports of preclinical work to show that electrical fields could potentially impair synaptic formation and neuronal connections affecting cognitive function on brains in development.

We therefore propose that TTF are a potential valuable treatment in this very small, but acutely sick, patient population. TTF should undergo further evaluation in clinical trials to confirm their benefit in children as observed in adult high-grade glioma.

\section{Financial \& competing interests disclosure}

$D A$ Bota received funding from Novocure clinical trial sponsorship and is a member of the Novocure speakers bureau. The authors have no other relevant affiliations or financial involvement with any organization or entity with a financial interest in or financial conflict with the subject matter or materials discussed in the manuscript apart from those disclosed.

No writing assistance was utilized in the production of this manuscript.

\section{Ethical conduct of research}

The authors state that they have obtained appropriate institutional review board approval or have followed the principles outlined in the Declaration of Helsinki for all human or animal experimental investigations. In addition, for investigations involving human subjects, informed consent has been obtained from the participants involved.

\section{Informed consent disclosure}

The authors state that they have obtained verbal and written informed consent from the patient/patients for the inclusion of their medical and treatment history within this case report.

\section{References}

Papers of special note have been highlighted as:

- of interest; $\bullet$ of considerable interest

1 Perry A, Miller CR, Gujrati M et al. Malignant gliomas with primitive neuroectodermal tumor-like components: a clinicopathologic and genetic study of 53 cases. Brain Pathol. 19(1), 81-90 (2009).

- Details the occurrence of CNS neoplasms with combined features of malignant glioma and primitive neuroectodermal tumor.

2 Sturm D, Orr BA, Toprak UH et al. New brain tumor entities emerge from molecular classification of CNS-PNETs. Cell 164(5), 1060-1072 (2016).

3 Fangusaro J. Pediatric high grade glioma: a review and update on tumor clinical characteristics and biology. Front. Oncol. 2, 105 (2012).

-. A comprehensive review of the epidemiology, diagnosis and treatment of pediatric high-grade glioma with an added focus on preclinical tumor models.

4 Mrugala MM, Engelhard HH, Dinh Tran D et al. Clinical practice experience with NovoTTF-100A system for glioblastoma: The Patient Registry Dataset (PRiDe). Semin. Oncol. 41(Suppl. 6), S4-S13 (2014).

-• Postmarketing registry of recurrent glioblastoma (GBM) patients who received NovoTTF ${ }^{\circledast}$ therapy in a real-world, clinical practice setting in the USA between 2011 and 2013.

5 Instructions for Use. NovoTTF-100A system. Novocure. Issue date: 12 May 2013. www.accessdata.fda.gov/
6 Lacouture ME, Davis ME, Elzinga G et al. Characterization and management of dermatologic adverse events with the NovoTTF-100A system, a novel anti-mitotic electric field device for the treatment of recurrent glioblastoma. Semin. Oncol. 41(Suppl. 4), S1-S14 (2014).

- Describes the dermatologic adverse events associated with the transducer scalp arrays of the tumor treating field (TTF) device while offering prophylactic and treatment-based strategies to better maximize adherence.

7 Huang RY, Rahman R, Ballman KV et al. The impact of T2/FLAIR evaluation per RANO criteria on response assessment of recurrent glioblastoma patients treated with bevacizumab. Clin. Cancer Res. 22(3), 575-581 (2016).

8 Rath P, Lal B, Ajala O et al. In vivo c-Met pathway inhibition depletes human glioma xenografts of tumor-propagating stem-like cells. Transl. Oncol. 6(2), 104-111 (2013).

9 Johnson KJ, Cullen J, Barnholtz-Sloan JS et al. Childhood brain tumor epidemiology: a brain tumor epidemiology consortium review. Cancer Epidemiol. Biomarkers Prev. 23(12), 2716-2736 (2014).

10 Paugh BS, Qu C, Jones C et al. Integrated molecular genetic profiling of pediatric high-grade gliomas reveals key differences with the adult disease. J. Clin. Oncol. 28(18), 3061-3068 (2010).

11 Jones C, Perryman L, Hargrave D. Paediatric and adult malignant glioma: close relatives or distant cousins? Nat. Rev. Clin. Oncol. 9(7), 400-413 (2012).
12 Sposto R, Ertel IJ, Jenkin RD et al. The effectiveness of chemotherapy for treatment of high grade astrocytoma in children: results of a randomized trial. A report from the Childrens' Cancer Study Group. J. Neurooncol. 7(2), 165-177 (1989).

13 Stupp R, Mason WP, Van Den Bent MJ et al. Radiotherapy plus concomitant and adjuvant temozolomide for glioblastoma. $N$. Engl. J. Med. 352(10), 987-996 (2005).

14 Chamberlain MC. Bevacizumab for the treatment of recurrent glioblastoma. Clin. Med. Insights Oncol. 5, 117-129 (2011).

15 Hoffman LM, Geller J, Leach J et al. A feasibility and randomized Phase II study of vorinostat, bevacizumab, or temozolomide during radiation followed by maintenance chemotherapy in newly-diagnosed pediatric high-grade glioma: Children's Oncology Group Study ACNS0822. Neuro Oncol. 17(Suppl. 3), iii39-iii40 (2015).

16 Johnston DL, Keene DL, Lafay-Cousin L et al. Supratentorial primitive neuroectodermal tumors: a Canadian pediatric brain tumor consortium report. J. Neurooncol. 86(1), 101-108 (2008).

17 Cohen KJ, Pollack IF, Zhou T et al. Temozolomide in the treatment of high-grade gliomas in children: a report from the Children's Oncology Group. Neuro Oncol. 13(3), 317-323 (2011).

18 Kirson ED, Schneiderman RS, Dbaly V et al. Chemotherapeutic treatment efficacy and sensitivity are increased by adjuvant alternating electric fields (TTFields). BMC Med. Phys. 9, 1 (2009). 
19 Stupp R, Wong ET, Kanner AA et al. NovoTTF-100A versus physician's choice chemotherapy in recurrent glioblastoma: a randomised Phase III trial of a novel treatment modality. Eur. J. Cancer 48(14), 2192-2202 (2012).

-• The first Phase III clinical trial comparing TTF monotherapy with chemotherapy in patients with recurrent GBM demonstrating comparable overall survival and improved quality of life.

20 Stupp R, Taillibert S, Kanner AA et al. Maintenance therapy with tumor-treating fields plus temozolomide vs temozolomide alone for glioblastoma: a randomized clinical trial. JAMA 314(23), 2535-2543 (2015).

-. A Phase III clinical trial and the second prospective study to demonstrate the efficacy of the NovoTTF device in GBM.
21 Giladi M, Schneiderman RS, Voloshin T et al. Mitotic spindle disruption by alternating electric fields leads to improper chromosome segregation and mitotic catastrophe in cancer cells. Sci. Rep. 5, 18046 (2015).

22 Turner SG, Gergel T, Wu H, Lacroix M, Toms SA. The effect of field strength on glioblastoma multiforme response in patients treated with the NovoTTF-100A system. World J. Surg. Oncol. 12, 162 (2014).

23 Hondroulis E, Melnick SJ, Zhang X, Wu ZZ, Li CZ. Electrical field manipulation of cancer cell behavior monitored by whole cell biosensing device. Biomed. Microdevices 15(4), 657-663 (2013).

24 Kanner AA, Wong ET, Villano JL, Ram Z, Investigators EF. Posthoc analyses of intention-to-treat population in Phase III comparison of NovoTTF-100A system versus best physician's choice chemotherapy. Semin. Oncol. 41(Suppl. 6), S25-S34 (2014).

25 Vymazal J, Wong ET. Response patterns of recurrent glioblastomas treated with tumor-treating fields. Semin. Oncol. 41(Suppl. 6), S14-S24 (2014).

-• Analyzes data from clinical trials to better characterize tumor response patterns compared with a kinetic model of tumor volume changes.

26 Aimone JB, Li Y, Lee SW, Clemenson GD, Deng W, Gage FH. Regulation and function of adult neurogenesis: from genes to cognition. Physiol. Rev. 94(4), 991-1026 (2014) 